\title{
Sur l'intégrale de Lebesgue-Stieltjes et les fonctions abso- lument continues de fonctions absolument continues
}

par M.lle Nrna Bart et M. D. Menchoff (à Moscon).

Nous nous proposons d'étudier dans ce Mémoire l'intégrale de LeBesGueSTIELTJEs d'une fonction mesurable $f(x)$ par rapport à une fonction $\varphi(x)$ absolument continue.

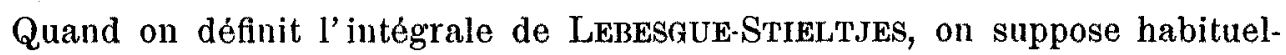
lement que la fonction $\varphi(x)$ est à variation bornée; on pose alors

$$
\varphi(x)=\varphi_{1}(x)-\varphi_{2}(x)
$$

chacune des fonctions $\varphi_{1}(x)$ et $\varphi_{2}(x)$ étant non décroissante; on définit l'intégrale de LEBESGUE-STIELTJES d' une fonction $f(x)$ par rapport à une fonction uon décroissante et l'on pose par définition

$$
\text { L. S. } \int_{a}^{b} f d \varphi=\text { L.S. } \int_{a}^{b} f(x) d \varphi_{1}-\text { L.S. } \int_{a}^{b} f(x) d \varphi_{2}\left({ }^{1}\right)
$$

Dans le présent Mémoire nous imposons à la fonction $\varphi(x)$ la condition d'être absolument continue, mais la définition de l'intégrale que nous considérons (\$1) est telle que l'intégrale

$$
\int_{a}^{b} f(x) d \varphi
$$

(1) M. Radon dans son Mémoire Theorie und Anwendungen der absolut additiven Men. genfunktionen (Sitzungsberichte der Kaiserlichen Akademie der Wissenschaften, Band 122, Abt. $I^{\mathrm{a}}$, s. $1205-1438$, Wien 1913) donne une définition très génerale de l'integrale de Strieltues, mais lui aussi ne considère que le cas où chacune des intégrales $\int_{a}^{b} f d \varphi_{1}$ et $\int_{a}^{b} f a \varphi_{2}$ existe et définit l'intégrale $\int_{a}^{b} f a \varphi$ comme leur différence. 
peut exister sans que les deux intégrales

$$
\int_{a}^{b} f(x) d \varphi_{1} \text { et } \int_{a}^{b} f(x) d \varphi_{2}
$$

existent.

En étudiant l'intégrale ainsi définie, nous voyons (\$3) qu'il y a des cas où l'intégrale définie $\int_{a}^{b} f(x) d \varphi$ existe, tandis que l'intégrale indéfinie $\int_{a}^{x} f(x) d \varphi$ n'existe pas quelque soit $x, a<x<b$. D'ailleurs, même quand cette intégrale est déterminée et fillie pour tout point $x, a \leq x \leq b$, elle peut représenter une fonction discontinue $(\$ 4)$.

On pourrait done croire qu' une telle définition de l'intégrale de LEBEsqueStieltues ne présente aucun intérêt. Mais il y a une classe remarquable de ces intégrales, ce sont les intégrales de la forme

$$
\text { L. } \mathrm{S} . \int_{a}^{x} f[\varphi(x)] d \varphi
$$

elles présentent une généralisation très naturelle des intégrales de M. LEBESGUE. On démontre $(\$ 6)$ que si l' intégrale indéfinie $\int_{a}^{\infty} f[\varphi(x)] d \varphi$ existe pour tout $x$, $a \leq x \leq b$, elle représente une fonction absolument continue de fonction absolument continue et reciproquement, toute fonction absolument continue de fonction absolument continue est (à une constante additive près) une intégrale de Lebesgue-Stieltjes de la forme $\int_{a}^{\infty} f[\varphi(x)] d \varphi$ (de même qu' une intégrale indéfinie de M. LebesGue est une fonction absolument continue et réciproquement).

Nous donnons ensuite $(\S 7)$ une. condition necessaire et suffisante pour $q u$ 'une fonction continue soit une fonction absolument continue de fonction absolument continue. On en déduit sans peine (\$9) que toute intégrale indéfinie de $M$. Denjoy est une fonction absolument continue de fonction absolument continue.

L'intégrale indéfinie de M. DenJoY a presque partout une dérivée déterminée et finie. Mais parmi les fonctions absolument continues de fonctions absolument continues, il y a des fonctions qui n'ont pas de dérivée sur un ensemble de mesure positive (\$9). 
On voit donc que cette classe de fonctions est beaucoup plus vaste que celle des fonctions absolument continues elle-mêmes. On pourrait croire que la classe des fonctions de la forme

$$
f\{\varphi[\psi(x)]\}
$$

$f, \varphi$ et $\psi$ étant absolument continues, présente une classe encore plus vaste. Nous démontrons (\$ 9) qu'il n'en est pas ainsi : cette classe coincide avec celles des fonctions absolument continues de fonctions absolument continues.

§ 1. Définition de l'intégrale de Lebesgue-Stieltjes. - Soit $f(x)$ une fonction mesurable et finie presque partout dans $(a . b)$ et $\varphi(x)$ une fonction absolument continue dans $(a, b)$.

Soit $\varepsilon$ un nombre positif et

$$
\ldots, l_{-n}, \ldots, l_{-1}, \quad l_{0}, l_{1}, \ldots, l_{n}, \ldots
$$

une échelle de nombres croissant de $-\infty \grave{a}+\infty$ par degrés $<\varepsilon$.

Désignons par $l_{n}$ l'ensemble des points pour lesquels on a

$$
l_{n-1} \leq f(x)<l_{n}
$$

et par $\lambda_{n}$ un nombre tel que

et formons la série

$$
l_{n-1} \leq \lambda_{n}<l_{n}
$$

$$
S=\sum_{n=-\infty}^{n=+\infty} \lambda_{n} \operatorname{var} \varphi(x)
$$

$\operatorname{var} \varphi(x)$ désignant la variation algébrique de $\varphi(x)$ sur l'ensemble $e_{n}\left(^{1}\right)$; $\boldsymbol{e}_{n}$

(1) Rappelons la definition de la vaviation algébrique (Ch. de la VALLÉE.Poussin, Cours d'Analyse Infinitésimale, t. 1, 30 édition, pag. 267):

Soit $F(x)$ une fonction continue of $E$ un ensemble mesurable. Enfermons $E$ en une infinité dénombrable d'intervalles $\left(a_{n}, b_{n}\right)$ sans points communs deux à deux et considérons la somme $\sum_{n=1}^{\infty}\left[F\left(b_{n}\right)-F\left(a_{n}\right)\right]$. Si cette série est absolument convergente, sa valeur est la variation de $F(x)$ dans l'ensemble des intervalles $\left(a_{n}, b_{n}\right)$. Si cette variation tend vers une limite toujours la même quand on fait tendre la somme $\mathbf{\Sigma}\left(b_{n}-a_{n}\right)$ des longueurs des intervalles vers la mesure de $E$, cette limite est la variation algébrique de $F(x)$ dans l'ensemble $\boldsymbol{E}$.

Il est important de remarquer que dans le cas que nons etudions, la variation de $\varphi(x)$ sur un ensemble peut être positise ou negative, puisqu' on ne suppose guère que la fonction $\varphi(x)$ soit toujours croissante on toujours décroissante. C'est par cette raison que l' intégrale de Lebesque-Streltues $\int_{a}^{b} f(x) d \varphi$ pent exister sans que les deux intégrales $\int_{a}^{b} f(x) d \varphi_{t}$ et $\int_{a}^{b} f(x) d \varphi_{2}$ existent, à̀ l'on a posé $\varphi(x)=\varphi_{1}(x)-\varphi_{2}(x), \varphi_{1}(x)$ et $\varphi_{2}(x)$ étant non décroissantes. 
supposons que cette série converge absolument et que sa somme $S$ tende vers une limite finie quand $\varepsilon$ tend vers zéro, cette limite étant toujours la même quel que soit le choix des nombres $l_{n}$ et $\lambda_{n}$; nous dirons alors que la fonction $f(x)$ est sommable par rapport $\dot{a} \varphi(x)$ et nous donnerons à cette limite le nom d'intégrale de Lebesgue-Stieltjes de la fonction $f(x)$ par' rappoirt à la fonction $\varphi(x)$

$$
\lim S=\text { L. S. } \int_{a}^{b} f(x) d \varphi .
$$

On sait (') que la variation d'une fonction absolument continue $\varphi(x)$ sur un ensemble mesurable $e$ est égale à

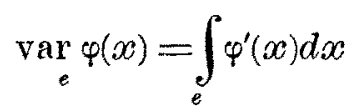

l' intégrale de cette formule étant une intégrale de M. Lebesaur.

La somme $S$ qui sert à définir l'intégrale de Lebesgue-STIELTJes devient alors

$$
S=\sum_{n=-\infty}^{n=+\infty} \lambda_{n} \int_{\varepsilon_{n}} \varphi^{\prime}(x) d x
$$

On voit qu' en posant $\varphi(x)=x$ on réduit la somme $S$ à la somme

$$
\sum_{n=-\infty}^{n=+\infty} \lambda_{n} \text { mes } e_{n}
$$

dont la limite (si elle existe) est l'intégrale de M. LEBESGUE de la fonction $f(x)$. La variation de $\varphi(x)$ joue donc dans l' intégrale de Lebesgue-Stieltjes le même rôle que la mesure dans l'intégrale de Lebesgue. On sait, que la valeur de l'intégrale de LEBESGUE n' est pas changée quand on modifie arbitrairement la fonction $f(x)$ sous le signe d'intégrale sur un ensemble de mesure nulle. Soit $E$ un ensemble où l'on a presque partout

$$
\varphi^{\prime}(x)=0 \text {. }
$$

La variation de $\varphi(x)$ est done nulle sur $E$ et sur chaque ensemble $E^{\prime \prime} \subset E$. Il suit de la définition même qu' en modifiant arbitrairement la fonction $f(x)$ sur $E$ on ne change pas la valour de l' intégrale de LeBesgue-STrELtJes $\int_{a}^{b} f(x) d \varphi$.

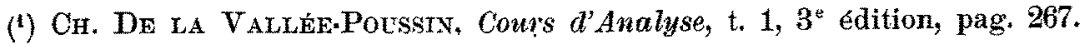


Ainsi les ensembles où l'on a presque partout $\varphi^{\prime}(x)=0$ jouent dans l'étude de l'intégrale de Lebesgue-Stieltjes le même rốle que les ensembles de mesure nulle dans l'intégrale de Lebesgue: il sont négligeables.

Cela posé, passons à l'étude de l'intégrale de LeBesgue-Strieltues.

§ 2. Comparaison avec l'intégrale de M. Lebesgue. - Nous allons démontrer d'abord le thèorème suivant.

THÉoRÈme 1. Si le produit $f(x) p^{\prime}(x)$ est sommable dans $(a, b)$, la fonction $f(x)$ est sommable par rapport $a \varphi(x)$ et l'on a

$$
\text { L. S. } \int_{a}^{x} f(x) d \varphi=\mathrm{L} . \int_{a}^{x} f(x) \varphi^{\prime}(x) d x . \quad a \leq x \leq b \text {. }
$$

En reprenant les nombres $l_{n}, \lambda_{n}$ et $e_{n}$ du $\S 1$ et en désignant par $e_{n}(x)$ la partie de l'ensemble $e_{n}$ située dans l'intervalle $(a, x)$, on voit que la somme $S$ qui sert à définir l'intégrale de Lebesgue-Stieltses peut être écrite sous la forme

$$
S(x)=\sum_{n=-\infty}^{n=+\infty} \lambda_{n} \int_{e_{n}(x)} \varphi^{\prime}(x) d x . \quad a \leq x \leq b .
$$

D'autre part, l'intégrale de LeBesgue du produit $f(x) \varphi^{\prime}(x)$ qui existe, d'après l'hypothèse faite, peut se présenter dans la forme d' une série

$$
S_{1}(x)=\sum_{n=-\infty}^{n=1} \int_{e_{4 l}(x)}^{\infty} f(x) \varphi^{\prime}(x) d x
$$

car les ensembles $e_{n}(x)$ n' ont pas de points communs et leur somme coïncide avec l'intervalle $(a, x)$. Le produit $f(x) \varphi^{\prime}(x)$ étant sommable, cette série est absolument convergente.

La différence des termes généraux de la série absolument convergente $S_{1}(x)$ et de la série $S(x)$ est égale à

$$
\int_{e_{i n}^{\prime}(x)}\left[f(x)-\lambda_{n}\right] \varphi^{\prime}(x) d x .
$$

Mais les valeurs de la fonction $f(x)$ dans l'ensemble $e_{n}$, donc à fortiori dans $e_{n}(x)$, appartiennent a l'intervalle $\left(l_{t-1}, l_{i}\right)$ de l'axe des $Y$ ainsi que la valeur $\lambda_{n}$; donc la différence $f(x)-\lambda_{n}$ ne surpasse pas en valeur absolue la longueur de cet intervalle, mais cette dernière est inférieure à un nombre 
positif donné $\varepsilon$. Par suite, la différence des termes généraux des séries $S_{1}(x)$ et $S(x)$ ne surpasse pas la valeur

$$
\varepsilon \int_{e_{n}(x)}\left|\varphi^{\prime}(x)\right| d x
$$

mais cette valeur est le terme général d'une série absolument convergente dont la somme est égale à

$$
\varepsilon \int_{a}^{x}\left|\varphi^{\prime}(x)\right| d x
$$

Il s'en suit que la série considérée $S(x)$ est aussi absolument convergente et que sa somme a pour limite l'intégrale de LeBESGue du produit $f(x) \varphi^{\prime}(x)$ dans $(a, x)$ quand $\varepsilon$ tend vers zéro. Nous avons donc démontré l'identité

$$
\text { L.S. } \int_{a}^{x} f(x) d \varphi=\text { L. } \int_{a}^{x} f(x) \varphi^{\prime}(x) d x . \quad \text { (c. q. f. d.). }
$$

Remar'que. Si la fonction $f(x)$ est bornée, le produit $f(x) \varphi^{\prime}(x)$ est sommable quelque soit la fonction absolument continue $\varphi(x)$. Il en suit qu' une fonction borne $f(x)$ est sommable par rapport $\dot{a}$ une fonction $\varphi(x)$ absolument continue arbitraire et son intégrale de Lebesgue-Stieltjes est égale à l' intégrale de Lebesgue du produit $f(x) \varphi^{\prime}(x)$.

§ 3. Dans le cas où le produit $f(x) \varphi^{\prime}(x)$ est sommable dans $(a, b)$, l'existence de l'intégrale définie $\int_{a}^{b} f(x) d \varphi$ n'est qu' un cas particulier de l'existence de l'intégrale indéfinie $\int_{a}^{x} f(x) d \varphi$ pour tout point $x, a \leq x \leq b$. Mais dans le cas où le produit $f(x) \varphi^{\prime}(x)$ n' est pas sommable, il peut arriver que l'intégrale définie existe tandis que l'intégrale indéfinie n'existe pas, et cette circonstance peut se présenter même pour une fonction $f(x)$ sommable.

Nous allons construire une fonction sommable $f(x)$ et une fonction absolument continue $\varphi(x)$ telles que l'intégrale

$$
\int_{i}^{1} f(x) d \varphi
$$


existe, tandis que l'intégrale indefinie $\int_{0}^{x} f(x) d \varphi$ n' existe pas quelque soit $x$, $0<x<1$.

Posons

$$
a_{n}=\frac{1}{n+2}, \quad b_{n}=\frac{n+1}{n+2} \quad(n=0,1,2,3, \ldots)
$$

Soit

$$
f(x)=\sqrt{n+2} \text { sur }\left(a_{n+1}, a_{n}\right) \text { et }\left(b_{n}, b_{n+1}\right) \quad(n=0,1,2,3, \ldots)
$$

On voit sans peine que la fonction $f(x)$ est sommable. En effet, la longueur $\mathrm{d}_{1}$. chacun des intervalles $\left(a_{n+1}, a_{n}\right)$ et $\left(b_{n}, b_{n+1}\right)$ est égale à

$$
\frac{1}{(n+2)(n+3)}
$$

On a done

$$
\sum_{n=0}^{\infty} \int_{a_{n+1}}^{a_{n}} f(x) d x=\sum_{n=0}^{\infty} \frac{\sqrt{n+2}}{(n+2)(n+3)}<\sum_{n=0}^{\infty} \frac{1}{(n+2)^{2}}
$$

et la dernière série converge. On a la même inégalité pour la somme des intégrales de $f(x)$ sur les intervalles $\left(b_{n}, b_{n+1}\right)$. Donc

$$
\text { L. } \int_{0}^{1} f(x) d x
$$

existe.

Soit maintenant

$$
\begin{array}{llll}
\psi(x)=+\sqrt{n+2} & \text { sur } & \left(a_{n+1}, a_{n}\right) & (n=0,1,2,3, \ldots) \\
\psi(x)=-\sqrt{n+2} & \text { sur } \quad\left(b_{n}, b_{n+1}\right) & (n=0,1,2,3, \ldots) .
\end{array}
$$

La fonction $\psi(x)$ est sommable puisque

$$
|\psi(x)|=f(x)
$$

et $f(x)$ est sommable. Soit

$$
\varphi(x)=\mathrm{L} \cdot \int_{0}^{x} \psi(x) d x
$$


La fonction $\varphi(x)$ est absolument continue. Il est clair que

L.S. $\int_{0}^{1} f(x) d \varphi=\sum_{n=0}^{\infty} \sqrt{n+2}\left(\mathrm{~L} \cdot \int_{a_{n+1}}^{a_{n}} \varphi^{\prime}(x) d x+\mathrm{L} \cdot \int_{b_{n}}^{b_{n+1}} \varphi^{\prime}(x) d x\right)=\sum_{n=1}^{\infty} \sqrt{n+2}\left(\int_{a_{n+1}}^{a_{n}} \psi(x) d x+\int_{b_{n}}^{b_{n+1}} \psi(x) d x\right)=$

$$
=\sum_{n=1}^{\infty}(n+2)\left[+\frac{1}{(n+2)(n+3)}-\frac{1}{(n+2)(n+3)}\right]=0 \text {. }
$$

L'intégrale définie L. S. $\int_{0}^{1} f(x) d \varphi$ existe donc et elle est égale à 0 .

Soit maintenant $x$ un point quelconque, $0<x<1$. Supposons d'abord qu' on ait pour un certain $n=n_{0}$

$$
a_{n_{0}+1} \leq x \leq a_{n_{0}} \quad\left(a_{0}=\frac{1}{2}, \text { donc } \quad x \leq \frac{1}{2}\right)
$$

On a alors

$$
\begin{aligned}
& \text { L. S. } \int_{0}^{x} f(x) d \varphi=\sum_{n=n_{0}+1}^{\infty} \text { L. S. } \int_{a_{n+1}}^{a_{n}} f(x) d \varphi+\text { L. S. } \int_{a_{n_{0}+1}}^{x} f(x) d \varphi= \\
& =\sum_{n=n_{0}+1}^{\infty} \sqrt{n+2} \operatorname{L} \cdot \int_{a_{n+1}}^{a_{n}} \psi(x) d x+\sqrt{n_{n}+2} \int_{a_{n_{0}+1}}^{x} \psi(x) d x \geq \sum_{n=n_{0}+1}^{\infty} \frac{\sqrt{n+2}}{(n+2)(n+3)}=\sum_{n=n_{0}+1}^{n+3} \frac{1}{n+2},
\end{aligned}
$$

la série étant divergente on voit que L. S. $\int_{0}^{x} f(x) d \varphi$ n' existe pas.

Dans le cas où l'on a pour un certain $n=n_{0}$

$$
b_{n_{0}} \leq x \leq b_{n_{0}+1} \quad\left(b_{0}=\frac{1}{2}, \text { donc } \quad x \geq \frac{1}{2}\right)
$$

on divise l'intégrale en deux parties:

$$
\text { L. S. } \int_{0}^{x} f(x) d \varphi=\text { L. S. } \int_{0}^{\frac{1}{2}-x} f(x) d \varphi+\int_{\frac{1}{2}-x}^{x} f(x d \varphi .
$$

La première de ces intégrales n'existe pas, car on peut lui appliquer le raisonnement précédent, $\frac{1}{2}-x$ étant nécessairement contenu dans un certain 
$\left(a_{n+1}, a_{n}\right)$; quant à la seconde intégrale, elle est égale à zéro; la démonstration est tout à fait analogue à la démonstration de l'égalité

$$
\int_{0}^{1} f(x) d \varphi=0
$$

On voit ainsi que l'intégrale indéfinie $\int_{0}^{x} f(x) d \varphi$ n' existe pas quel que soit $x, 0<x<1$.

(c. q. f. d.).

\& 4. On peut se poser la question suivante: en supposant que l'intégrale indéfinie de LebesGue-Stieltues est déterminée et finie en chaque point de l'intervalle $(a, b)$, peut-on affirmer qu' elle représente une fonction continue? Nous allons répondre négativement a cette question en définissant une fonclion sommable $f(x)$ et une fonction $\varphi(x)$ absolument continue telles que l' intégrale indefinie

$$
\mathfrak{F}(x)=\mathrm{L} . \mathrm{S} \cdot \int_{0}^{x} f(x) r l \varphi
$$

existe pour tout point $x, 0 \leq x \leq 1$ el sa valeur est partout finie, mais $\mathfrak{F}(x)$ est discontinue au point $x=1$.

Posons

$$
a_{n}=1-\frac{1}{n^{2}} \quad(n=1,2,3, \ldots)
$$

Soit

$$
f(x)=n^{\frac{3}{2}} \text { sur }\left(a_{n}, a_{n+1}\right) \quad(n=1,2,3, \ldots) .
$$

On voit que $f(x)$ est sommable puisque la série

$$
\sum_{n=1}^{\infty} \int_{a_{n}}^{a_{n+1}} f(x) d x=\sum_{n=1}^{\infty} n^{\frac{3}{2}} \frac{2 n+1}{n^{2}(n+1)^{2}}
$$

converge. Soit

$$
\begin{array}{lll}
\psi(x)=+n^{\frac{3}{2}} & \text { sur }\left(a_{n}, \frac{a_{n}+a_{n+1}}{2}\right)
\end{array} \quad(n=1,2,3, \ldots) .
$$


Puisque $|\psi(x)|=f(x)$, la fonction $\psi(x)$ est sommable. Posons

$$
\varphi(x)=\mathrm{L} \cdot \int_{u}^{x} \psi(x) d x
$$

$\varphi(x)$ est donc absolument continue. Il est évident que

L. S. $\int_{0}^{1} f(x) d \varphi=\sum_{n=1}^{\infty} n^{\frac{3}{2}}\left(\int_{a_{n}}^{\frac{a_{n+a_{n+1}}}{2}} \psi(x) d x+\int_{\frac{a_{n+1}+a_{n+1}}{2}}^{2} a_{n+1}^{a_{n+1}}(x) d x\right)=\sum_{n=1}^{\infty} n^{3}\left(\frac{1}{2} \frac{2 n+1}{n^{2}(n+1)^{2}}-\frac{1}{2} \frac{2 n+1}{n^{2}(n+1)^{2}}\right)=0$.

L'intégrale définie $\int_{0}^{1} f(x) d x$ existe donc et elle est égale à 0.

Soit maintenant $x$ un point quelconque $0 \leq x<1$. L'intégrale indéfinie

$$
\int_{0}^{x} f(x) d \varphi
$$

existe puisque $f(x)$ est bornée sur le segment $(0, x)$ et nous avons vu $(\S 2)$ $q u^{\prime}$ une fonction bornée est sommable par rapport à une fonction $\varphi(x)$ absolument continue arbitraire. Mais on a quelque soit $n$

$$
\begin{aligned}
\mathfrak{J}\left(\frac{a_{n}+a_{n+1}}{2}\right) & =\mathrm{L} \cdot \mathrm{S} \cdot \int_{0}^{\frac{a_{n+a_{n+1}}}{2}} f(x) d \varphi=\mathrm{L} . \mathrm{S} \cdot \int_{0}^{a_{n}} f(x) d \varphi+\mathrm{L} \cdot \mathrm{S} \cdot \int_{a_{n}}^{\frac{a_{n+a_{n+1}}}{2}} f(x) d \varphi= \\
& =\sum_{k=0}^{n-1} k^{\frac{3}{2}}\left(\int_{a_{n}}^{\frac{a_{k+a_{k+1}}}{2}} \psi(x) d x+\int_{a_{n+1}}^{a_{k+1}} \psi(x) d x\right)+n^{\frac{3}{2}} \int_{a_{n}}^{\frac{a_{n+1}}{2}} \psi(x) d x= \\
& =\sum_{k=0}^{n-1} k^{3}\left[\frac{1}{2} \frac{2 k+1}{k^{2}(k+1)^{2}}-\frac{1}{2} \frac{2 k+1}{k^{2}(k+1)^{2}}\right]+n^{3} \frac{1}{2} \frac{2 n+1}{n^{2}(n+1)^{2}}=\frac{n(2 n+1)}{2(n+1)^{3}}
\end{aligned}
$$

Quand $n$ tend vers l'infini, on a

$$
\lim _{n \rightarrow \infty} \frac{a_{n}+a_{n+1}}{2}=1
$$


et

$$
\lim _{n \rightarrow \infty} \mathscr{F}\left(\frac{a_{n}+a_{n+1}}{2}\right)=\lim _{n \rightarrow \infty} \frac{n(2 n+1)}{2(n+1)^{2}}=1
$$

mais $\mathfrak{F}(1)=0, \mathfrak{F}(x)$ est done discontinue au point $x=1$

(c. q. f. d.).

$\S 5$. Nous avons vu au $\$ 4$ que l'intégrale indéfinie de LeBESGUE-STIELTJEs (supposée déterminée et finie en chaque point d' un intervalle) peut avoir des points de discontinuité. Nous allons démontrer maintenant que si l'intégrale indefinie de Lebesgue-Stieltjes existe en chaque point $x, a \leq x \leq b$, elle est ou bien continue, on bien une limite de fonctions continues (c' est ì dire une fonction de classe 1 au plus d'après la classification de M. BAIRE).

Sans restreindre la généralité des considérations on peut supposer que la fonction $f(x)$ ne prend que des valeur's entières. En effet, en désignant par $E[A]$ le plus grand entier contenu dans le nombre réel $A$, nous voyons qu' on peut diviser $f(x)$ en une somme de deux fonctions

$$
f(x)=E[f(x)]+\theta(x)
$$

dont la première est une fonction mesurable qui n'a que des valeurs entières et la seconde est une fonction mesurable et bonne, puisque $0 \leq \theta(x)<1$.

D'après la remarque faite à la fin du $\S 2$, la fonction $\theta(x)$ est donc sommable par rapport à une fonction absolument continue $\varphi(x)$ absolument quelconque et son intégrale indéfinie de LEBEsGuE-STIELTJEs coincide avec l'intègrale de Lebesgue du produit $\theta(x) \varphi^{\prime}(x)$, il représente donc une fonction absolument continue. D' autre part il est evident que le théorème \& l'intégrale d'une somme est égale a la somme des intégrales \$ subsiste pour les intégrales de Lebesque-STieltues. Done il nous suffit de considérer des fonctions qui ne prennent que des valeurs entières.

Soit $e_{n}$ l'ensemble des points où l'on a

$$
f(x)=n, \quad(n=0, \pm 1, \pm 2, \ldots)
$$

et $\Psi_{n}(x)$ une fonction égale à $\varphi^{\prime}(x)$ dans $e_{n}$ et à zéro en dehors de $e_{n}$

On a alors

$$
(n=0, \pm 1, \pm 2, \ldots)
$$

$$
\text { L. S. } \int_{a}^{x} f(x) d \varphi=\sum_{n=-\infty}^{n=+\infty} n \int_{a}^{x} \psi_{n}(x) d x
$$


la derniére série étant absolument convergente quel que soit $x$, puisque nous avons supposé que l'intégrale indéfinie de LEBEsGun-STIELTJES est déterminée et finie partout dans $(a, b)$. Le terme général de cette série, étant une intégrale indéfinie de M. Lebesgue multipliée par $n$, est donc une fonction continue et l'on voit ainsi que l'intégrale indéfinie de Lebesque-StrentJes est la limite de fonctions continues, ce qui prouve la proposition énoncée. (c. q. f. d.).

\section{§ 6. Un cas remarquable d'intégrabilité au sens de Lebesgue-Stieltjes. -} Nous avons vu aux paragraphes précédents que l'intégrale indéfinie L. S. $\int_{a}^{a} f(x) d \varphi$ $n^{\prime}$ est pas nécessairement déterminée dans $a \leq x \leq b$ si l'intégrale définie $\int_{a}^{b} f(x) d \varphi$ existe, et quand elle est déterminée et finie, elle est généralennent discontinue.

Considérons maintenant le cas où la fonction sous le signe d'intégrale est une fonction de la fonction $\varphi(x)$, c' est-à-dire considérons les intégrales de la forme

$$
\text { L. S. } \int_{a}^{x} f[\varphi(x)] d \varphi .
$$

Nous verrons que si les extrêmés absolus de $\varphi(x)$ sont aux bornes de l'intervalle $(a, b)$, l'intégrale indéfinie existe pour tout point $x, a \leq x \leq b$ sous la seule condition d'existence de l'intégrale définie $\int_{a}^{b} f[\varphi(x)] d \varphi$; d'ailleurs, chaque fois que l'intégrale de la forme $\int_{a}^{x} f[\varphi(x)] d \varphi$ existe pour tout $x, a \leq x \leq b$, elle représente une fonction continue dans $(a, b)$. Nous allons étudier maintenant les intégrales de cette forme, mais nous avons besoin de quelques définitions et lemmes préliminaires.

Un ensemble de points $e$, situé dans $(a, b)$, sera dit complet par rapport à une fonction $\varphi(x)$, si l'on a toujours

$$
\varphi\left(x^{\prime}\right) \neq \varphi\left(x^{\prime \prime}\right)
$$

pour tout couple de points $x^{\prime}$ et $x^{\prime \prime}$ tels que $x^{\prime}$ appartient à $e$, et $x^{\prime \prime}$ à son complémentaire $C e$ par rapport a l'intervalle $(a, b)$.

LEMME 1. Soit $\varphi(x)$ une fonction absolument continue definie dans un intervalle $(a, b)$, e un ensemble complet par rapport $\dot{a} \varphi(x)$, \& l'ensemble 
des valeurs de $\varphi(x)$ sur e, enfin $\mathcal{B}^{*}$ la partie de $\mathcal{E}$ comprise entre les points $\varphi(a)$ et $\varphi(b)$. La variation de $\varphi(x)$ sur $e$ est alors égale en valeur. absolue à la mesure de $\mathcal{G}^{*}$ et elle a le signe de la diffèrence $\varphi(b)-\varphi(a)$.

En effet, soit $f(u)$ une fonction égale à 1 sur $\mathscr{G}$ et à zéro en dehors de $\mathcal{G}$. On voit de suite qu' en posant $A=\varphi(a)$ et $B=\varphi(b)$ on a

$$
\operatorname{mes} \mathcal{g}^{*}= \pm \mathrm{L} \cdot \int_{A}^{B} f(u) d u
$$

le signe + devant l'intégrale correspond au cas $A<B$ et le signe - au cas $B<A$. La fonction $f(u)$ est une fonction bornée. Par conséquent la régle d'intégration par substitution pour les intégrales de LEBEsGue s'applique ('); on trouve done en posant $u=\varphi(x)$

$$
\operatorname{mes} \mathcal{G}^{*}= \pm \mathrm{L} \cdot \int_{A}^{B} f(u) d u= \pm \mathrm{L} \cdot \int_{a}^{b} f\left[\varphi(x) \mid \varphi^{\prime}(x) d x\right.
$$

Si le point $x$ appartient à $e, \varphi(x)$ appartient à $\mathcal{E}$ (d'aprés la définition de $\mathfrak{E}$ ), donc $f[\varphi(x)]=1$. L' ensemble $e$ étant complet par hypothése, $\varphi(x)$ ne peut appartenir à $\mathscr{G}$ si $x<C e$, donc pour tout point $x<C e$, on a $f[\varphi(x)]=0$. Il s'en suit

$$
\int_{a}^{b} f[\varphi(x)] \varphi^{\prime}(x) d x=\int_{e} 1 \cdot \varphi^{\prime}(x) d x+\int_{C_{e}} 0 \cdot \varphi^{\prime}(x) d x=\int_{e} \varphi^{\prime}(x) d x=\operatorname{var}_{e} \varphi(x) .
$$

On a douc

Plus précisément

$$
\operatorname{mes} \mathcal{G}^{*}= \pm \underset{e}{\operatorname{var} \varphi(x)}
$$

$$
\begin{aligned}
& \operatorname{var} \varphi(x)=+\operatorname{mes} \mathcal{G}^{*} \text { quand } A<B, \text { donc } \varphi(a)<\varphi(b)
\end{aligned}
$$

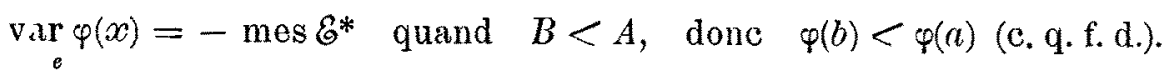

On déduit de ce lemme le théorème suivant:

Théorìme 2. Pour que l'intégrale

$$
\text { L. S. } \int_{a}^{b} f[\varphi(x)] d \varphi
$$

(') Ch. De la Vallée-Poussin, Cours d'Analyse, t. 1, 3édition, pag. 283. 
existe, il est necessaire et suffisant que la fonction $r$ soit sommable entree les limites $A=\varphi(a)$ el $B=\varphi(b)$; on a alors

$$
\text { L. S. } \int_{a}^{b} f[\varphi(x)] d \varphi=\mathrm{L} \cdot \int_{A}^{B} f(u) d u \text {. }
$$

Supposons d'abord que l'intégrale $\int_{a}^{b} f[\varphi(x)] d \varphi$ existe. Divisons l'axe des $y$ par une échelle de nombres $l_{n}(n=0, \pm 1, \pm 2, \ldots)$ croissant de $-\infty$ à $+\infty$ par degrés $<\varepsilon$; soit $e_{n}$ l'ensemble de tous les points $x$ de l'intervalle $(a, b)$ pour lesquels on a

$$
l_{n-1} \leq f[\varphi(x)]<l_{n}
$$

on a d'après la definition même de l'intégrale de LEBESGUe-STIELTJES

$$
\text { L. S. } \int_{a}^{b} f[\varphi(x)] d \varphi=\lim \sum_{n=-\infty}^{n=+\infty} \lambda_{n} \operatorname{var} \varphi(x)
$$

$\lambda_{n}$ étant un nombre compris entre $l_{n-1}$ et $l_{n}$ et la limite étant prise dans l'hypothèse que toutes les différences $l_{n}-l_{n-1}$ tendent uniformément vers zéro d'une manière quelconque. Cette limite existe, puisque l'intégrale L.S. $\int_{a}^{b} f[\varphi(x)] d \varphi$ existe per hypothèse.

Soit $\mathscr{G}_{n}$ l'ensemble des valeurs de $\varphi(x)$ sur $e_{n}$ et $\mathcal{G}^{*}{ }_{n}$ la partie de $\mathfrak{C}_{n}$ comprise entre les points $A=\varphi(a)$ et $B=\varphi(b)$. Chacun des ensembles $e_{n}$ $(n=0, \pm 1, \pm 2, \ldots)$ est complet par rapport à la fonction $\varphi(x)$; en effet si l'on avait

$$
\varphi\left(x^{\prime}\right)=p\left(x^{\prime \prime}\right)
$$

$x^{\prime}$ appartenant à $e_{n}$ et $x^{\prime \prime}$ à son complémentaire par rapport à $(a, b)$, on aurait aussi

$$
f\left[\varphi\left(x^{\prime}\right)\right]=f\left[\varphi\left(x^{\prime \prime}\right)\right]
$$

done

$$
l_{n-1} \leq f\left[\varphi\left(x^{\prime \prime}\right)\right]<l_{n},
$$

et $x^{\prime \prime}$ appartiendrait aussi à $e_{n}$, ce qui est impossible puisqu'il doit appartenir à son complémentaire. 
Tous les ensembles $e_{n}$ étant complets par rapport à $\varphi(x)$, le lemme sur la variation s' applique et l'on a

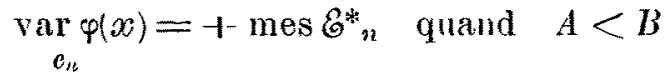

$$
\begin{aligned}
& \underset{e_{n}}{\operatorname{var}_{n} \varphi(x)=-\operatorname{mes} \mathscr{G}^{*}{ }_{n}} \quad \text { quand } \quad A>B \quad(n=0, \pm 1, \pm 2, \ldots) .
\end{aligned}
$$

On a done

$$
\text { L. S. } \int_{a}^{b} f[\varphi(x)] d \varphi= \pm \lim _{n=-\infty} \sum_{n=+\infty}^{n=+\infty} \lambda_{n} \text { mes } \mathcal{E}^{*}{ }_{n} \quad\left(\begin{array}{l}
B-A>0 \\
B-A<0
\end{array}\right) .
$$

D'autre part, d'après la définition même de $\mathscr{G}^{*} n$, on voit que $\mathrm{c}^{\prime}$ est l'ensemble de toutes les valeurs de $u$ entre $A$ et $B$, pour lesquelles on a

$$
l_{n-1} \leq f(u)<l_{n}
$$

il s'en suit, d'aprés la définition même de l'intégrale de M. LeBEsGue,

$$
\pm \lim \sum_{n=-\infty}^{n=+\infty} \lambda_{n} \operatorname{mes} \mathcal{G}^{*}{ }_{n}=\int_{\boldsymbol{A}}^{B} f(u) d u
$$

on a donc

$$
\text { L. S. } \int_{a}^{b} f[\varphi(x)] d \varphi=\mathrm{L} \cdot \int_{A}^{B} f(u) d u \text {. }
$$

La premiére partie du théorème est ainsi démontrée.

Réciproquement, si $f(u)$ est sommable dans $(A, B)$, l' intégrale de LEBESGUE entre les limites $A$ et $B$ existe; on a

$$
\text { L. } \int_{\mathbb{A}}^{B} f(u) d u= \pm \lim \sum_{n=-\infty}^{n=+\infty} \lambda_{n} \text { mes } \mathcal{E}_{*}^{*}, \quad\left(\begin{array}{l}
+ \text { correspond au cas } B>A \\
- \text { correspond au cas } B<A
\end{array}\right)
$$

où l'on a désigné par $\mathcal{G}^{*}{ }_{n} \mathrm{l}^{\prime}$ ensemble des points $u$ entre $A$ et $B$ pour lesquels on a

$$
l_{n-1} \leq f(u)<l_{n},
$$

et par $\lambda_{n}$ un point tel que

$$
l_{n-1} \leq \lambda_{n}<l_{n} .
$$

Mais d'après le lemme 1 , on a

$$
\pm \operatorname{mes} \mathcal{E}^{*}{ }_{n}=\operatorname{var}_{e_{n}} \varphi(x) \quad(n=0, \pm 1, \pm 2, \ldots)
$$


$e_{n}$ étant l'ensemble de tous les points $x$ entre $a$ et $b$ pour lesquels on a

$$
l_{n-1} \leq f[\varphi(x)]<l_{n} .
$$

Il s' en suit, d'après la définition de l'intégrale de LEBEsGue-STIEL'TJES, que

$$
\mathrm{L} . \int_{j}^{B} f(u) d u= \pm \lim \sum_{n=-\infty}^{n=+\infty} \lambda_{n} \text { mes } \mathfrak{G}_{n}{ }^{*}=\lim \sum_{n=-\infty}^{n=+\infty} \lambda_{n} \operatorname{var}_{e_{n}} \varphi(x)=\mathrm{L} . \mathrm{S} \cdot \int_{a}^{b} f[\varphi(x)] d \varphi
$$

et l' existence de l'intégrale de Lebesgue-Stieltjes est ainsi établie (c. q. f. d.).

On déduit de ce théorème les corollaires suivants:

Corollaire 1. Pour que l'intégrale

$$
\text { L. S. } \int_{a}^{x} f[\varphi(x)] d \varphi
$$

existe pour tout $x, a \leq x \leq b$, il faut et il suffit que $f(u)$ soit sommable dans l'intervalle $(m, M), m$ étant le minimum et $M$ le maximum de $u=\varphi(x)$ dans $(a, b)$; on a alors

$$
\text { L. S. } \int_{a}^{x} f[\varphi(x)] d \varphi=\mathrm{L} \cdot \int_{\boldsymbol{A}}^{u} f(u) d u
$$

oiu $A=\varphi(a)$ et $u=\varphi(x)$.

En effet, soit $a^{\prime}$ un point de $(a, b)$ tel que $\varphi\left(a^{\prime}\right)=m$ et $b^{\prime}$ un point de $(a, b)$ tel que $\varphi\left(b^{\prime}\right)=M$ ). Si l'intégrale de Lebesque-StrieltJes existe pour tout point $x$, l'intégrale $\int_{a^{\prime}}^{b^{\prime}} f[\varphi(x)] d \varphi$ existe nécessairement et $\mathrm{d}^{\prime}$ après le théorème 2 il en suit que $f(u)$ est sommable entre $m=\varphi\left(a^{\prime}\right)$ et $M=\varphi\left(b^{\prime}\right)$.

Réciproquement, si $f(u)$ est sommable dans $(m, M)$, soit $x$ un point ques: conque de $(a, b)$ et $u=\varphi(x), u$ est alors compris dans $(m, M)$, puisque toutes les valeurs de $\varphi(x)$ dans $(\alpha, b)$ appartiennent à $(n, M)$, et d'ailleurs L. $\int_{\boldsymbol{A}}^{u} f(u) d u$ existe. Il s'en suit, d'après le théorème 2 que $\int_{a}^{x} f[\varphi(x)] d \varphi$ existe, et $x$ étaut quelconque, le corollaire 1 est démontré (l'égalité des valeurs des intégrales de Lebesgue et de Lebesgue-Stieltues suit aussi du théoreme 2). 
Corollatre 2. Si les extrêmés absolus de $\varphi(x)$ sont aux bornes de l'intervalle $(a, b)$ el si l'intégrale

$$
\text { L. S. } \int_{a}^{b} f[\varphi(x)] d \varphi
$$

existe, l'intégrale indéfnie

$$
\text { L. S. } \int_{a}^{x} f[\varphi(x)] d \varphi
$$

existe pour tout $x, a \leq x \leq b$.

En effet, les valeurs $M$ et $m$ du corollaire 1 coincident dans le cas considéré avec $B=\varphi(b)$ et $A=\varphi(a)$. Puisque l'integrale

$$
\text { L. S. } \int_{a}^{b} f[\varphi(x)] d \varphi
$$

existe par hypothèse, il en suit que $f(u)$ est sommable dans $(A, B)$, donc entre le minimum et le maximum de $\varphi(x)$ dans $(a, b)$, ce qui entraine (corollaire 1) l'existence de l'intégrale indéfinie

$$
\text { L.S. } \int_{a}^{\infty} f[\varphi(x)] d \varphi
$$

pour tout point $x, a \leq x \leq b$.

ThÉonème 3. Si l'integrale indefinie

$$
\text { L. S. } \int_{a}^{x} f[\varphi(x)] d \varphi
$$

existe pour tout point $x, a \leq x \leq b$, elle represente une fonction absolument continue de fonction absolument continue. Réciproquement, toute fonction absolument continue de fonction absolument continue est (à une constanle additive près) une intègrale indéfinie de. Lebesgue-Stieltjes de cette forme.

En effet, d'après le corollaire 1 , si cette intẻgrale indéfinie existe pour tout $x, f(u)$ est sommable dans $(m, M)$ et l'on a

$$
\text { L. S. } \int_{a}^{x} f[\varphi(x)] d \varphi=\mathrm{L} \cdot \int_{A}^{u} f(u) d u=F(u)-F(A)=F[\varphi(x)]-F[\varphi(a)],
$$


oú l'on a désigné par $F(u)$ l'intégrale indéfinie de $f(u)$ (au sens de M. LebesGUE). Il en suit que $F(u)$ est absolument continue; $\varphi(x)$ étant absolument continue par hypothèse, la première partie du théorème 3 est ainsi démontrée.

Supposons maintenant que $\mathfrak{S}(x)$ soit une fonction de la forme

$$
\mathfrak{F}(x)=F[\varphi(x)]
$$

$\varphi(x)$ étant absolument continue dans un intervalle $(a, b)$ et $F(u)$ absolument continue dans ( $m, M), m$ et $M$ étant respectivement le minimum et le maximum de $\varphi(x)$ dans $(a, b)$.

Soit $A=\varphi(a)$; toute fonction absolument continue étant à une constante additive prés l'intégrale indéfinie de M. LEBESGUE d'une fonction sommable, on peut écrire

$$
F(u) \equiv F(A)+\mathrm{L} \cdot \int_{\boldsymbol{A}}^{u} f(u) d u
$$

en désignant par $f(u)$ la dérivée de $F(16)$ qui est nécessairement sommable.

Mais nous savons (corollaire 1) que l'existence de l'intégrale indéfinie

$$
\mathrm{L} \cdot \int_{4}^{u} f(u) d u
$$

entraîne celle de l'intégrale indéfinie

$$
\text { L. S. } \int_{a}^{x} f[\varphi(x)] d \varphi
$$

et l'égalité des valeurs de ces intégrales. Il en suit que l'on a

$$
\mathscr{F}(x)=F[\varphi(x)]=F(u)=F[\varphi(\alpha)]+\int_{a}^{x} f[\varphi(x)] d \varphi
$$

et le théorème 3 est ainsi complètement démontré.

(c. q. f. d.).

On voit donc que la classe des intégrales indéfinies de LEBESGUE-STIELTJES de la forme $\int_{a}^{x} f[\varphi(x)] d \varphi$ coüncide avec celle des fonctions absolument continues de fonctions absolument continues, de même que la classe des intégrales indéfinies de M. Lebesgue conncide avec celle des fonctions absolument continues. 
On peut poursuivre cette analogie en considérant les dérivées des intégrales étudiées. On sait que l'intégrale indéfinie de M. LEBESGue a toujours une dérivée, presque partout déterminée, finie et égale à la fonction intégrée. Nous avons remarqué au $\S 1$ que dans l'étude des intégrales de LEBEsGUeSTIELtJEs on peut considérer comme * ensembles de mesure nulle * les ensembles où l'on a presque partout

$$
\varphi^{\prime}(x)=0 .
$$

Il est donc naturel de se poser la question si la dérivée de l'intégrale indéfinie

$$
\mathscr{H}(x)=\mathrm{L} . \mathrm{S} \cdot \int_{a}^{x} f[\varphi(x)] d \varphi
$$

existe presque en tous les points où l'on a

$$
\varphi^{\prime}(x) \neq 0 \text {. }
$$

Il est aisé de voir que la réponse est affirmative. On a le théorème:

ThÉorème 4. L'intègrale indéfnie (supposée existante pour tout $x$ $a \leq x \leq b)$

$$
\mathscr{f}(x)=\text { L.S. } \int_{a}^{x} f[\varphi(x)] d \varphi
$$

a une dèrivèe $\mathfrak{F}^{\prime}(x)$ déterminèe et finie presque en tous les points $x$ de l'ensemble $R$ où la dérivée $\varphi^{\prime}(x)$ existe et l'on a

$$
\varphi^{\prime}(x) \neq 0
$$

et cette dèrivèe vèrifie l'égalité

$$
\mathfrak{g}^{\prime}(x)=\int[\varphi(x)] \varphi^{\prime}(x)
$$

En effet, d'aprés le théorème 3 , on a

$$
\mathfrak{F}(x)=F[\varphi(x)]-F[\varphi(a)] .
$$

D'aprés la régle de différentiation d'une fonction de fonction, $\mathfrak{F}^{\prime}(x)$ est égale à

$$
F^{\prime}(\varphi) \varphi^{\prime}(x)
$$

en tout point oủ ces deux dérivées sont déterminées et finies. 
Soit $\mathscr{G}$ l'ensemble de tous les points $u$ tels que $F^{\prime}(u)$ n'existe pas on n'est pas égale à $f(u)$. On a évidemment

$$
\text { mes } \mathfrak{B}=0
$$

Soit $E$ l'ensemble de tous les points $x$, tels que les valeurs de $\varphi(x)$ en ces points appartiemuent à $\mathfrak{G}$. Nous allons démontrer que la partie commune des ensembles $R$ et $E$ est un ensemble de mesure nulle. En effet, $\varphi(x)$ étant absolument continue et la mesure de l'ensemble $\mathcal{E}$ de ses valeurs sur $E$ étant nulle, on a d'aprés un théorème de M. CH. DE LA VALléE-Poussin ( $\left.{ }^{1}\right)$ le résultat suivant: on bien mes $E=0$, on bien la dérivée $\varphi^{\prime}(x)$ est nulle presque en tous les points de $E$. Dans les deux cas, l'ensemble des points de $E$ où la dèrivée $\varphi^{\prime}(x)$ existe et n' est pas nulle est un ensemble de mesure nulle. Il en suit que la partie commune de $E$ et de $R$ est un ensemble de mesure nulle.

Soit $x$ un point de $R$ n'appartenant pas à $E ; \varphi^{\prime}(x)$ est déterminée et finie et $u=\varphi(x)$ n' appartient pas à $\mathcal{E}$, donc $F^{\prime}(u)=f(u)=f[\varphi(x)]$; on a donc

$$
\mathfrak{F}^{\prime}(x)=f[\varphi(x)] \varphi^{\prime}(x),
$$

cette égalité a donc lieu presque en tous les points de $R$.

(c. q. f. d.).

On voit donc que si l'intégrale indéfinie de Lebesgue-Stieltjes de la forme $\int_{a}^{x} f[\varphi(x)] d \varphi$ existe pour tout point $x$, elle a une dérivée partout sauf sur un ensemble où la dérivée $\varphi^{\prime}(x)$ est presque partout nulle, donc partout en dehors d'un ensemble qui est négligeable dans l'étude de l'intégrale de LEBESGUE-STIELTJES. D' ailleurs cette dérivée est égale à la fonction intégrée multipliée par $\varphi^{\prime}(x)$. Le multiplicateur $\varphi^{\prime}(x)$ est remplacé par l'unité pour les intégrales de M. LEBESGUE, ce qui est naturel car nous avons vu (\$1) que l'intégrale de LeBesque-Stieltues se réduit à celle de M. Lebesgue quand on pose $\varphi(x)=x$, donc $\varphi^{\prime}(x)=1$.

§ 7. Propriété caractéristique des fonctions absolument continues de fonctions absolument continues. - $\mathscr{F}(x)$ étant une fonction continue, quelle est la condition nécessaire et suffisante pour qu'elle soit une intégrale de LebesGue-Stieltues de la forme $\int_{a}^{x} f[\varphi(x) d \varphi$, ou, ce qui est le même, pour

(1) Ch. De ra Vallépolssin, Con's.d.Analyse, t. I, 3 e Edition, pag. 281-283. 
qu' elle soit une fonction absolument continue de fonction absolument continue?

Pour répondre à cette question, nous avons besoin de quelques lemmes préliminaires.

LeMme 2. Soit $\mathfrak{F}(x)$ une fonction continue, $E$ un ensemble de points, tel que $\left|\mathfrak{F}^{\prime}(x)\right|<M$ sur $E$ ( $M$ constante) et $\mathfrak{G}$ l'ensemble des valeur's de $\mathfrak{F}(x)$ sur E. On a

$$
\text { mes } \mathfrak{G} \leq 2 M \text { mes } E \text {. }
$$

Soit $\xi$ un point de $E$ et $\eta$ un nombre positif aussi petit qu' on veut.. Soit $\delta$ un intervalle contenant $\xi$; pourvu que $\delta$ soit assez petit, on a pour tout point $x$ de $\delta$

$$
\left|\frac{\mathfrak{F}(x)-\mathfrak{H}(\xi)}{x-\xi}\right|<M+\eta
$$

$\mathfrak{F}(x)$ étant continue et $\omega_{\delta}$ l'oscillation de $\mathfrak{F}(x)$ sur $\delta$, on a

$$
\omega_{\delta}=\mathfrak{F}\left(x^{\prime \prime}\right)-\mathfrak{H}\left(x^{\prime}\right)
$$

$x^{\prime \prime}$ et $x^{\prime}$ étant deux points de $\delta$; on a donc

$$
\left|\frac{\mathfrak{F}\left(x^{\prime \prime}\right)-\mathfrak{g}(\xi)}{x^{\prime \prime}-\xi}\right|<M+\eta ; \quad\left|\frac{\mathfrak{H}\left(x^{\prime}\right)-\mathfrak{F}(\xi)}{x^{\prime}-\xi}\right|<M+\eta
$$

Il en suit

$$
\omega_{\mathcal{E}} \leq\left|\mathfrak{F}\left(x^{\prime \prime}\right)-\mathfrak{F}(\xi)\right|+\left|\mathfrak{F}(\xi)-\mathfrak{F}\left(x^{\prime}\right)\right|<(M+\eta)\left|x^{\prime \prime}-\xi\right|+(M+\gamma)\left|x^{\prime}-\xi\right|
$$

mais puisque $x^{\prime}$ et $x^{\prime \prime}$ appartiennent à $\delta$ ainsi que le point $\xi$, on a

$$
\omega_{\delta}<2(M+\eta) \delta .
$$

Soit $E_{n}$ l'ensemble de tous les point $\xi$ tels que l'inégalité précédente est vérifiée pour tout intervalle $\delta$ contenant $\xi$ et de longueur $\leq \frac{1}{n}$ et $\mathcal{E}_{n}$ l' ensemble des valeurs de $\mathfrak{F}(x)$ sur $E_{n}$. Supposons que le kemme soit démontré pour l'ensemble $E_{n}(n=1,2,3, \ldots)$ et démontrons qu'il l'est alors pour l'ensemble $E$.

En effet, $E$ est la somme des $E_{n} ;$ done $\mathscr{E}$ la somme des $\mathscr{G}_{n}$. D'aillenrs on a

$$
\mathfrak{G}_{1} \subset \mathfrak{G}_{2} \subset \ldots \subset \mathfrak{G}_{n} \subset \ldots
$$


On peut donc, quelque petit que soit $\sigma$, choisir l'entier $n$ assez grand pour que l'on ait

$$
\text { mes } \mathcal{G}-\operatorname{mes} \mathfrak{G}_{n}<\sigma .
$$

En supposant que le lemme soit déja démontré pour l'ensemble $E_{n}$, on a

$$
\text { mes } \mathcal{G}-\sigma<\operatorname{mes} \mathcal{G}_{n} \leq 2 M \text { mes } E_{n} \leq 2 M \text { mes } E
$$

et $\sigma$ étant aussi petit qu' on vent, il en suit

$$
\text { mes } \mathscr{B} \leq 2 M \text { mes } E \text {. }
$$

Il suffit donc de démontrer le lemme pour l'ensemble $E_{n}$.

Enfermons l'ensemble $E_{n}$ en une infinité dénombrable d'intervalles sans points communs deux a deux

tels qu' on ait

$$
\delta_{1}, \delta_{2}, \ldots, \delta_{n}, \ldots
$$

$$
\sum_{n=1}^{\infty} \delta_{n}<\operatorname{mes} E_{n}+\varepsilon
$$

$\varepsilon>0$ étant aussi petit qu' on veut. Il est évident qu' on peut toujours supposer que chacun des intervalles $\delta_{n}$ est de longueur $\leq \frac{1}{n}$ et qu'il contient nécessairement au moins un point $\xi$ de $E_{n}$.

En désignant par $\omega_{k}$ l'oscillation de $\mathfrak{F}(x)$ sur l'intervalle $\delta_{k}$, on a donc l'inégalité

Il en suit

$$
\omega_{k}<2(M+\eta) \delta_{k} .
$$

$$
\sum_{k=1}^{\infty} \omega_{k}<2(M+\eta) \sum_{k=1}^{\infty} \delta_{k}<2(M+\eta)\left(\text { mes } E_{n}+\varepsilon\right) .
$$

Il en résulte que l'ensemble $\mathcal{B}_{n}$ des valeurs de $\mathfrak{F}(x)$ sur $E_{n}$ est contenu dans un système d'intervalles dont la longueur totale est inférieure à $2(M+\eta)\left(\operatorname{mes} E_{n}+\varepsilon\right)$ et puisque $\varepsilon$ et $\eta$ sont aussi petits qu'on veut, on a

$$
\text { mes } \mathfrak{G}_{n} \leq 2 M \operatorname{mes} E_{n} \text {. (c. q. f. d.). }
$$

Corollaike 1. $\mathfrak{F}(x)$ ètant une fonction continue dont la dérivée $\mathfrak{F}^{\prime}(x)$ est délerminè el finie sur un ensemble $E$ de nesure nulle, l'ensemble $\mathscr{6}$ des valeu's de $\mathfrak{F}(x)$ sur $E$ esi un ensemble de mesure nulle.

En effet, on peut écrire

$$
E=\sum_{n=1}^{\infty} E_{n}
$$


$E_{n}$ étant la partie de $E$ où la dérivée $\mathfrak{F}^{\prime}(\mathrm{x})$ vérifie l'inégalité

$$
\left|\mathfrak{g}^{\prime}(x)\right|<n . \quad(n=1,2,3, \ldots) .
$$

Chacun des ensembles $E_{n}$ est de mesure nulle. Soit $\mathcal{E}_{n}$ l'ensemble des valeurs de $\mathfrak{F}(x)$ sur $E_{1 .}$. En appliquant le lemme 2 , on a

$$
\operatorname{mes} \mathcal{G}_{n} \leq 2 n \operatorname{mes} E_{n}=0 . \quad(n=1,2,3, \ldots) .
$$

L'ensemble $\mathcal{E}$ des valeurs de $\mathfrak{F}(x)$ sur $E$ étant contenu dans la somme $\sum_{n=1}^{\infty} \mathcal{E}_{n}$ est donc aussi un esemble de mesure nulle.

Conollatre 2. $\mathfrak{F}(x)$ étant une fonction continue dont la dèrivede $\mathfrak{F}^{\prime}(x)=0$ sur un ensemble $E$, l'ensemble $\mathcal{G}$ des valeur's de $\mathfrak{F}(x)$ sur $E$ est un ensemble de mesure nulle.

On applique le lemme 2 en tenant compte du fait que $M$ est maintenant aussi petit qu'on veut. La mesure de $\mathcal{E}$ étant aussi petite qu' on veut est nécessairement nulle.

(c. q. f. d.).

Definilion. Nous dirons avec M. Lusin (') qu' une fonction continue $\mathfrak{F}(x)$ jouit al la propriele $N$ dans un intervalle $(a, b)$ si l'ensemble des valeurs de $\mathscr{f}(x)$ sur chaque ensemble de mesure nulle situé dans $(a, b)$ (et d'ailleurs absolument quelconque) est nécessairement un ensemble de mesure nulle.

Lemme 3. Soit $\varphi(x)$ une fonction continue jouissant de la proprielé $N$ et ayant presque partout une deriveje $\varphi^{\prime}(x)$ qui est une fonction sommable. La fonction $\varphi(x)$ est alors absolument continue.

Cette proposition a été démontrée par M. Menchoff dans un autre recueil $\left({ }^{2}\right)$, c' est pourquoi nous omettons ici sa démonstration.

LeMme 4 , Soit $u=\psi(y)$ une fonction absolument continue toujours croissante (ou loujours decroissante) et telle que $\psi^{\prime}(y) \neq 0$ presque partout; la fonction inver'se $y=F(u)$ est alor's absolument continue.

(') N. Lusin, L'intégrale et la série trigonométrique (en russe), Moscou, 1915, pag. 109.

(') D. Menchowr, Sur la représentatîn conforme des domaines plans, Mathematische Annalen, Band 95, Heft 5, pag. 645. 
Soit $e$ un ensemble de points situé sur l'axe des $y$, mes $e>0$. Supposons que la relation $u=\psi(y)$ lui fasse correspondre un ensemble $e^{\prime}$ de l'axe des $u$ tel que mes $e^{\prime}=0$. Puisque $\psi(y)$ est absolument continue par hypothèse il en suit ( $\left.{ }^{\prime}\right)$ que $\psi^{\prime}(y)=0$ presque partout sur $e$, ce qui contredit à la condition du lemme. Il correspond done à chaque ensemble $e$ situé sur l'axe des $y$ et de mesure positive un ensemble $e^{\prime}$ de l'axe des $u$, dont la mesure est encore positive. En particulier, il correspond à deux points $y_{1}$ et $y_{2}, y_{1} \neq y_{2}$ deux points $u_{1}$ et $u_{2}, u_{1} \neq u_{2}$. La fonction inverse $y=F(u)$ est donc définie d'une manière univoque. Elle est continue et croissante (décroissante) puisqu'il en est ainsi pour $\Psi(y)$. Elle a donc presque partout une dérivée et cette dérivée est sommable. Il suffit donc (en vertu du lemme 3) de démontrer qu' elle jouit de la propriété $N$. Mais cela est évident. En effet, soit $e^{\prime}$ un ensemble de mesure nulle de l'axe des $u$. L'ensemble $e$ des valeurs de $y=F(u)$ sur $e^{\prime}$ est nécessairement de mesure nulle, car si l'on avait mes $e>0$, on aurait aussi mes $e^{\prime}>0$, comme nous avons vu au commencement de la démonstration.

(c. q. f. d.).

LEMme 5. Soit $\varphi(x)$ une fonction continue dans un intervalle $(a, b)$. Supposons que sa dérivée $\varphi^{\prime}(x)$ vérifie l'inégalité $\left|\varphi^{\prime}(x)\right|<M$ (M constanle absolue) sur un ensemble $\mathfrak{T}$ et que l'ensemble $\mathscr{N}$ des valeur's de $\varphi(x)$ sur le complémentaire $C \mathfrak{K}$ de $\mathfrak{K}$ est un ensemble de mesure nulle. Dans ces conditions, $\varphi(x)$ est absolument continue dans $(a, b)$.

En effet, soit $x_{1}$ et $x_{2}$ deux points quelconques de $(a, b)$; soit $x_{1}<x_{2}$. Considérons l'intervalle

$$
\delta=\left(x_{1}, x_{2}\right)
$$

L'ensemble des valeurs de $\varphi(x)$ sur $\delta$ est la somme de l'ensemble des valeurs de $\varphi(x)$ sur la partie $\mathfrak{K}_{\delta}$ de $\mathscr{N}$ appartenant à $\delta$ et de l'ensemble des valeurs de $\varphi(x)$ sur la partie $C \mathscr{T K} \delta$ de $C \mathscr{V} \mathcal{K}$ appartenant $\dot{a} \delta$. La mesure du premier de ces ensembles est (d'après le lemme 2) $\leq 2 M$ mes $\mathfrak{K}_{\delta}$, donc a fortiori $\leq 2 M \delta$ et le second, étant une partie de $\mathscr{T}$, est nécessairement de mesure nulle. On a donc

$$
\omega_{\delta} \leq 2 M \delta
$$

$\omega_{\delta}$ étant l'oscillation de $\varphi(x)$ sur $\delta$. On en conclut

$$
\left|\varphi\left(x_{1}\right)-\varphi\left(x_{2}\right)\right| \leq 2 M \delta=2 M\left|x_{1}-x_{2}\right| ;
$$

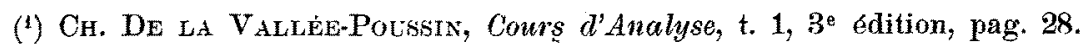


$x_{1}$ et $x_{2}$ étant deux points quelconques de $(a, b), l^{\prime}$ inégalité précédente est donc la condition bien connue de Lipschitz et $\varphi(x)$ vérifiant la condition de LIPSCHITZ est nécessairement absolument continue.

(c. q. f. d.).

Ces préliminaires terminés, nous pouvons démontrer le théorème fondamental suivant.

ThÉoRÈm $5\left({ }^{1}\right)$. Soit $\mathfrak{F}(x)$ une fonction continue, $E$ l'ensemble de tous les points où la dérivée $\mathscr{G}^{\prime}(x)$ n'existe pas ou n'est pas finie et $\mathcal{E}$ l'ensemble des valeur's de $\mathfrak{F}(x)$ sur $E$. La condition nécessaire et suffisante pour que $\mathfrak{F}(x)$ soit une fonction absolument continue de fonction absolument continue est que l'on ail mes $\mathcal{E}=0$.

1) La condition est nécessaire. La fonction $\mathscr{F}(x)$ étant une fonction absolument continue de fonction absolurent continue, on a

$$
\left.\mathfrak{F}(x)=l^{\prime} \mid \varphi(x)\right]
$$

$F(u)$ et $\varphi(x)$ étant des fonctions absolument continues.

Soit $e^{\prime}$ l'ensernble de tous les points $x$ tels que $\varphi^{\prime}(x)$ n'existe pas on n' est pas finie et $\mathcal{G}^{\prime} l^{\prime}$ ensemble des valeurs de $\varphi(x)$ sur $e^{\prime}$. En vertu de la continuité absolue de $\varphi(x)$ on a

$$
\operatorname{mes} e^{\prime}=0
$$

et par conséquent (une fonction absolument continue jouit toujours de la proprièté $N$ )

$$
\text { mes } \mathfrak{G}^{\prime}=0 \text {. }
$$

Soit $\mathscr{G}^{\prime \prime}$ l'ensemble de tous les points $u$ tels que $F^{\prime}(u)$ n'existe pas ou n' est pas finie; $F(u)$ étant absolument continue, on a

$$
\operatorname{mes} \mathcal{G}^{\prime \prime}=0 \text {. }
$$

Soit enfin $e^{\prime \prime}$ l' ensemble de tous les points $x$ tels que les valeurs de $\varphi(x)$ dans ces points appartiennent à $\mathcal{G}^{\prime \prime}$. Il est aisé de voir que l'ensemble $E$ est contenu dans la somme $e^{\prime}+e^{\prime \prime}$

$$
E \subset e^{\prime}+e^{\prime \prime}
$$

(on a désigné par $E$ l'ensemble des points où la dérivée $\mathfrak{F}^{\prime}(x)$ n'existe pas

(1) Nous venons d'apprendre que M. ZARETsky a obtenu récemment et indépendamment le même résultat; ce résultat n' avait pas encore été publié. 
ou n'est pas finie). En effet, soit $x_{0}$ un point qui n'appartient pas à $e^{\prime}+e^{\prime \prime}$ et $u_{0}=\varphi\left(x_{0}\right)$. On a alors, d'après la règle de differentiation d'une fonction de fonction,

$$
\mathfrak{H}^{\prime}\left(x_{0}\right)=F^{\prime \prime}\left(u_{0}\right) \varphi^{\prime}\left(x_{0}\right)
$$

chacune des dérivées $F^{\prime}\left(u_{0}\right)$ et $\varphi^{\prime}\left(x_{0}\right)$ étant déterminée et finie, $\mathfrak{F}^{\prime}\left(x_{0}\right)$ l'est aussi, done $x_{0}$ n' appartient pas à $E$.

Il en suit que l'ensemblo $\mathcal{E}$ des valeurs de $\mathfrak{F}(x)$ sur $E$ est contenu dans l'ensemble des valeurs de $\mathscr{F}(x)$ sur $e^{t}+e^{\prime \prime}$ et nous allons voir que cet ensemble est de mesure nulle. En effet, l'ensemble des valeurs de $\mathscr{F}(x)$ sur $e^{\prime}+e^{\prime \prime}$ coincide avec l'ensemble des valeurs de $F(u)$ sur $\mathscr{G}^{\prime}+\mathcal{E}^{\prime \prime}$; mais nous avons vu que mes $\mathscr{G}^{\prime}=$ mes $\mathscr{G}^{\prime \prime}=0$, donc en vertu de la continuité absolue de $F(u)$, l'ensemble de ses valeurs sur $\mathfrak{G}^{\prime}+\mathcal{G}^{\prime \prime}$ est un ensemble de mesure nulle.

(c. q. f. d).

2) La condition est suffisante. Supposons que la fonction $\mathscr{F}(x)$ soit définie dans un intervalle $(a, b)$. Soit $\mu$ le minimé et $M$ le maximé de $\mathfrak{F}(x)$ dans $(a, b)$. Si la fonction $\mathscr{f}(x)$ est une constante, le théorème à démontrer devient trivial. Supposons donc qu'il n'en soit pas ainsi et, par conséquent, $\mu<M$.

En désignant par $E$ l'ensemble de tous les points de $(a, b)$ où la dérivée $\mathfrak{F}^{\prime}(x)$ n'existe pas ou n'est pas finie et par $\mathscr{G}$ l'ensemble des valeurs de $\mathscr{F}(x)$ sur $E$, on a par hypothèse,

$$
\operatorname{mes} \mathfrak{g}=0
$$

Soit $e$ l'ensemble de tous les points où l'on a $\mathfrak{F}^{\prime}(x)=0$. L'ensemble des valeurs de $\mathfrak{F}(x)$ sur $e$ est un ensemble $e^{\prime}$; d'après le corollaire 2 du lemme 2 on a mes $e^{\prime}=0$. Soit $\mathcal{G}^{\prime}=\mathfrak{G}+e^{\prime}$. On a alors mes $\mathfrak{G}^{\prime}=0$, puisque mes $\mathscr{B}=0$ par hypothèse.

Soit $y_{0}$ un point fixe n' appartenant pas a $\mathcal{B}^{\prime}, \mu \leq y_{0} \leq M$. Pour tous les points $x$, tels que $\mathfrak{F}(x)=y_{0}$ la dérivée $\mathfrak{F}^{\prime}(x)$ existe, est finie et diffère de zéro. La droite $y=y_{0}$ coupe la courbe $y=\mathfrak{F}(x)$ en un nombre fini de points. En effet, $s^{\prime}$ il $y$ en avait une infinité, ils auraient au moins un point limite d'abscisse $x_{0}$ (d'après le principe de Bolzano-Weierstrass). En ce point $x_{n}$ la dérivée $\mathfrak{F}^{\prime}\left(x_{0}\right)$ serait indéterminée ou nulle, ce qui est impossible puisque $y_{n}$ n'appartient pas à $\mathcal{G}^{\prime}$, donc $x_{0}$ n' appartient ni à $E$ ni à $e$. Il correspond donc a chaque point $y$ n'appartenant pas à $\mathcal{B}^{\prime}$ un nombre fini de points $x$, tels que $\mathfrak{F}(x)=y$. 
Définissons une fonction $B(y)$ pour tous les points $y$ n'appartenant pas à $\mathcal{G}^{\prime}$ par la condition

$$
B(y)=\max \left|\mathfrak{F}^{\prime}(x)\right|
$$

pour tous les $x$ tel que $\mathfrak{f}(x)=y$.

La fonction $B(y)$ est ainsi définie presque partout dans $(\mu, M)$; elle est finie et essentiellement positive partout où elle existe, done presque partout dans $(\mu, M)$ et il est aisé de voir qu' elle est mesurable.

Posons

$$
\begin{array}{lll}
A(y)=\frac{1}{B(y)} & \text { si } & B(y) \geq 1 \\
A(y)=1 & \text { si } & B(y)<1 .
\end{array}
$$

La fonction $A(y)$ est ainsi définie presque partout sur $(\mu, M)$, elle est mesurable, finie et essentiellement positive partout ou elle existe, donc presque partcut (puisqu'il en est ainsi pour $B(y))$; enfin elle est bornée, car il suit de sa définition même qu'on a

$$
0<A(y) \leq 1 \quad \mu \leq y \leq M .
$$

Il en suit qu' elle est sommable dans $(\mu, M)$. Posons

$$
u=\psi(y)=\int_{\mu}^{y} A(y) d y
$$

et

$$
\varphi(x)=\psi(y)=\psi[\mathfrak{F}(x)]
$$

Nous allons démontrer que la fonction $\varphi(x)$ est absolument continue.

Soit $R^{\prime}$ l'ensemble de tous les points $y$ tels que la dérivée $\psi^{\prime}(y)$ n'existe pas, n'est pas finie ou ne vérifie pas l'égalitè

On a alors

$$
\psi^{\prime}(y)=A(y)
$$

$$
\operatorname{mes} R^{\prime}=0
$$

Soit $R$ l'ensemble de tous les points $x$ tels que les valeurs de $\mathscr{F}(x)$ dans ces points appartiennent à $R^{\prime}$.

Considérons un point $x_{0}$, qui n'appartient ni à $R$ ni à $E$. Nous allons démontrer que la dérivée $\varphi^{\prime}\left(x_{0}\right)$ existe et est Inférieure à 1 en valeur absolue. 
En effet, $x_{0}$ n'appartenant ni à $E$ ni à $R, \mathfrak{F}^{\prime}\left(x_{0}\right)$ existe, $\psi^{\prime}\left(y_{0}\right)$ existe aussi est elle est égale à $A\left(y_{0}\right)$, on a donc

$$
\begin{aligned}
\varphi^{\prime}\left(x_{0}\right)=A\left(y_{0}\right) \mathscr{F}^{\prime}\left(x_{0}\right) & =\frac{\mathfrak{F}^{\prime}\left(x_{0}\right)}{B\left(y_{0}\right)} \text { quand } \quad B\left(y_{0}\right) \geq 1 \\
& =\mathscr{F}^{\prime}\left(x_{0}\right) \text { quand } \quad B\left(y_{0}\right)<1
\end{aligned}
$$

Mais $B\left(y_{0}\right)$ et le maximum des valeurs absolue de $\left|\mathfrak{F}^{\prime}(x)\right|$ pour tous les points $x$, tels que $y_{0}=\mathfrak{F}(x)$. Il en suit

$$
B\left(y_{0}\right) \geq\left|\mathfrak{F}^{\prime}\left(x_{0}\right)\right|
$$

et l'on a donc dans les deux cas $\left[B\left(y_{0}\right) \geq 1\right.$ et $\left.B\left(y_{0}\right)<1\right]$

$$
\left|\varphi^{\prime}\left(x_{0}\right)\right| \leq 1
$$

On déduit de cette inégalité la continuité absolue de $\varphi(x)$ de la manière suivante.

Soit $\mathscr{K}$ l'ensemble des points de l'intervalle $(a, b)$ n'appartenant ni à $E$ ni à $R$. L'ensemble des valeurs de $\varphi(x)$ sur le complémentaire $C \mathscr{O K}$ de $\mathfrak{O K}$ coincide avec l'ensemble des valeurs de $\psi(y)$ sur la somme $\mathfrak{E}+R$, et puisque mes $\mathcal{G}=$ mes $R^{\prime}=0$ et $\psi(y)$ est absolument continue, cet ensemble est de mesure nulle. On applique alors le lemme 5 et l'on voit que $\varphi(x)$ est une fonction absolument continue (et d'ailleurs à nombres dérivés bornés).

Mais on a

$$
\varphi(x)=\psi[\mathscr{f}(x)] .
$$

La fonction $u=\psi(y)$ est absolument continue; on a

$$
\psi^{\prime}(y)=A(y)>0
$$

presque partout; $\psi(y)$ est donc croissante. Toutes les conditions du lemme 4 étant vérifiées, on en conclut que la fonction inverse

$$
y=F(u)
$$

est une fonction absolument continue et croissante. On a ainsi

$$
y=\mathfrak{F}(x)=F(u)=F[\mathfrak{f}(x)]
$$

et la fonction $\mathfrak{F}(x)$ est une fonction absolument continue de fonctiou absolument continue.

(c. q. f. d.). 
Remarque. Il suit de la démonstration du théorème que toute fonction absolument continue de fonction absolument continue peut être présentée dans la forme

$$
\mathfrak{F}(x)=F[\varphi(x)]
$$

$F$ et $\varphi$ étant absolument continues et d'ailleurs $F$ croissante et $\varphi$ a nombres dérivés bornés.

\& 8. Autres propriétés caractéristiques des fonctions absolument continues de fonctions absolument continues. - On peut indiquer encore deux propriétés caractéristiques de ces fonctious.

Nous dirons avec M. BANACH $\left(^{1}\right) q u^{\prime}$ une fonction continue $\mathfrak{F}(x)$ jouit de la propriete $\left(T_{1}\right)$, si l'eusemble des valeurs que la fonction $\mathscr{F}(x)$ prend une infinité de fois est de mesure nulle.

On peut démontrer que pour qu'une fonction continue $\mathscr{F}(x)$ soit une fonction absolument continue de fonction absolument continue il faut et il sufft qu' elle jouisse des propriétés $(N)$ et $\left(T_{1}\right)$.

Nous omettons la démonstration qui est donnée d' ailleurs par M. ${ }^{116} \mathrm{~N}$. BARY dans un autre recueil $\left({ }^{2}\right)$.

Enfin, M. Banach a démontré $\left({ }^{3}\right)$ que l'ensemble des propriétés $(N)$ et $\left(T_{1}\right)$ est équivalent à la condition $(S)$. On dit avec M. BANaCH $\left(^{*}\right) q u^{\prime}$ une fonction continue $\mathfrak{F}(x)$ satisfait $a \dot{~ l a ~ c o n d i t i o n ~(S) ~ l o r s q u ' a ̀ ~ c h a q u e ~ n o m b r e ~} \varepsilon>0$ correspond un nombre $\eta>0$ de manière que l'inégalite mes $_{\theta} E<\eta$ entraîne mes $_{\mathrm{e}} E_{y}<\varepsilon$, en désignant par $E_{y}^{\prime}$ l' ensemble des valeurs de $\mathfrak{F}(x)$ sur $E$ et par mes $_{e}$ la mesure extérieure. On peut donc énoncer une troisième propriété caractéristique de la classe étudiée :

Pour qu' une fonction continue $\mathfrak{F}(x)$ soit une fonction absolument continue de fonction absolument continue, il faut et il suffit qu' elle vérifie la condition (S).

\$ 9. La classe des fonctions absolument continues de fonctionø absolument continues. - La condition nécessaire et suffisante démontrée au $\S 7$ nous permet d'apprécier la grandeur de la classe des fonctions absolument con-

(1) S. BaNAcH, Sur une classe de fonctions continues, Fundam. Math., t. 8, 1926, pag. 166-172.

(2) N. BARY, Sur la représentation analytique d'une classe de fonctions continues. Comptes Rendus, t. 183, pag, 469.

(3) Loc. cit.

(4) Loe. cit. 
tinues de fonctions absolument continues. On déduit du théorème 5 les corollaires suivants :

CoRollaine 1. Toute fonction continue $\mathfrak{F}(x)$ qui jouit de la propriété $\mathrm{N}$ et dont la derivee $\mathfrak{F}^{\prime}(x)$ est presque partout déterminée et finie est une fonction absolument continue de fonction absolument continue.

En effet, d'après I'hypothèse faite sur $\mathfrak{F}(x)$, l'ensemble $E$ des points $x$ où la dérivée $\mathfrak{G}^{\prime}(x)$ n'existe pas oì n' est pas finie est un ensemble de mesure nulle. Nais puisque $\mathfrak{F}(x)$ jouit de la propriété $N$, l'ensemble $\mathscr{G}$ des valeurs de $\mathfrak{F}(x)$ sur $E$ est aussi un ensemble de mesure nulle. Il suit donc du théoréme précédemment démontré que $\mathscr{f}(x)$ est une fonction absolument continue de fonction absolument continue.

(c. q. f. d.).

Corollarre 2. L'integrale undefinie de M. Denjoy est une fonction absolument continue de fonction absolument continue.

Ėn effet, l'intégrale indéfinie de M. DEnJoY a presque partout une dérivée déterminée et finie. Elle jouit d'ailleurs de la propriété $N\left({ }^{1}\right)$. Le corollaire 2 suit don: immédiatement du corollaire 1.

(c. q. f. d.).

No: a avons vu (\$6) que l'intégrale indéfinie de Lebesgue-Strelirys de la form. $\int f(\varphi) d \varphi$ est une fonction absolument continue de fonction absolument continue et inversement, toute fonction absolument continue de fonction absolument continue est une intégrale de Lebesque-StieltJes de la forme $\int f(\varphi) d \varphi$. Le théorème 5 (et ccux du \& 8) nous font maintenant connaitre complètement la structure de cette intégrale et le corollaire 2 nous montre que la classe des intégrales indéfinies de M. Densor est contenue dans celle des intégrales de Lebesque-Sineltues de la forme $\int f(\varphi) d \varphi$.

Mais il n'en est ainsi que pour les intégrales de M. DenJoy au sens strict $\left({ }^{2}\right)$ : quant a celles de M. M. DenJoY-Khintchine $\left({ }^{3}\right)$, nous allons voir bientôt qu'il existe parmi ces intégrales des fonctions qui ne sont pas des fonctions absolument continues de fonctions absolument continues.

(1) X. Lusin, L'integrale et la série trigonométrique, pag. 116 et 118 .

(2) A. Densoy, Comptes Rendus, t. 154, pag. 859 et 1075.

(3) .1. Dexjoy, Annales de l'École Normale supérieure, vol. 33, pag. 127 et vol. 34, pag. 181 ; A. Kuxpchne, Comptes Rendus, t, 162, pag. 287. 
L'intégrale de M. DenJoy (au sens strict) a presque partout une dérivée déterminèe et finie. Mais il existe, quelque petit que soit $\varepsilon$, des fonctions absolument continues de fonctions absolument continues qui n'ont pas de dérivée sur un ensemble de mesure $1-\varepsilon$.

Pour construire une telle fonction, considérons l'intervalle $(0,1)$; enlevons de cet intervalle un intervalle concentrique de longueur $\frac{\varepsilon}{2}$, puis de chacun des segments restés un intervalle concentrique de longueur $\frac{\varepsilon}{2 \cdot 4}$, à la $h$-ième opération eulevons des $2^{k-1}$ segments restés des intervalles concentriques de longueur $\frac{\varepsilon}{2 \cdot 4^{k-1}}$ et ainsi de suite infiniment. Soit $\delta_{1}^{(k)}, \delta_{2}^{(k)}, \ldots \delta_{2^{k-1}}^{(k)}$ ' les intervalles enlevés à ha $k$-ième opération $; c_{1}^{(k)}, c_{2}^{(k)}, \ldots c_{2^{k-1}}^{(h)}$ leurs centres et $f_{1}^{(l)}$, $\rho_{2}^{(k)}, \ldots \rho_{2^{k}}^{(k)}$ les segments restés. On a $\left({ }^{1}\right)$

$$
\begin{aligned}
\sum_{i=1}^{2^{k}} p_{i}^{(l)} & =1-\sum_{s=1}^{k} \sum_{i=1}^{2^{s-1}} \delta_{i}^{(s)}=1-\frac{\varepsilon}{2} \sum_{s=1}^{k} \sum_{i=1}^{2^{s-1}} \frac{1}{4^{s-1}}=1-\frac{\varepsilon}{2} \sum_{s=1}^{k} 2^{s-1} 4^{s-1}= \\
& =1-\frac{\varepsilon}{2}\left(\frac{1-\frac{1}{2^{k}}}{1-\frac{1}{2}}\right)=1-\varepsilon\left(1-\frac{1}{2^{k}}\right)
\end{aligned}
$$

donc

$$
P_{i}^{(k)}=\frac{1-\varepsilon\left(1-\frac{1}{2^{k}}\right)}{2^{k}} \quad\left(\begin{array}{l}
i=1,2, \ldots 2^{k} \\
k=1,2,3, \ldots
\end{array}\right) .
$$

L'ensemble parfait $P$ qu' on obtient en enlevant de $(0,1)$ tous ces $\delta_{i}^{(k)}$ $\left(i=1,2, \ldots 2^{k-1}, k=1,2, \ldots\right)$ est un ensemble de mesure $1-\varepsilon$. Soit $\mathscr{f}(x)$ une fonction définje par les conditions

$$
\begin{array}{rlll}
\mathfrak{J}(x)=0 & \text { pour } & x \subset P \\
\mathscr{F}(x)=2^{3 k}\left(x-a_{i}^{(k)}\right)\left(b_{i}^{(k)}-x\right) & \text { sur } & \delta_{i}^{(k)}=\left(a_{i}^{(k)}, b_{i}^{(k)}\right) & \left(\begin{array}{l}
i=1,2, \ldots 2^{k-1} \\
k=1,2,3, \ldots
\end{array}\right) .
\end{array}
$$

Il en suit que

$$
\mathfrak{F}\left(C_{i}^{(k)}\right)=2^{3 k} \stackrel{\substack{\delta(k) \\ 2}}{{ }^{-}} \frac{\delta_{i}^{(k)}}{2}=2^{3 k} \frac{\varepsilon}{4^{k}} \frac{\varepsilon}{4^{k}}=2^{3 k} \frac{\varepsilon^{2}}{4^{2 k}}=\frac{\varepsilon^{2}}{2^{k}}
$$

(1) En désignant par une même lettre un intervalle et sa lor,gueur. 
$\mathscr{F}(x)$ est une fonction continue; elle a une dérivée déterminée et finie pour tout point $x$ qui est intérieur à l'un des intervalles contigus de l'ensemble parfait $P$. Nous allons démontrer qu' elle n'a pas de dérivée pour tout point $x$ de l'ensemble $P$.

On a

$$
\frac{\mathfrak{J}(x+h)-\mathfrak{F}(x)}{h}=\frac{\mathfrak{F}(x+h)}{h} \text { pour } \quad x \subset P .
$$

Si le point $x+h$ tend vers le point $x$ en restant toujours sur $P$, on a

$$
\lim _{h \rightarrow 0} \frac{\mathfrak{H}(x+h)}{h}=0 .
$$

Mais le point $x+h$ peut tendre vers le point $x$ en restant toujours dans un intervalle contigu à $P$. Le point $x$ étant un point de $P$ appartient à une infinité de segments $\rho_{i}^{(k)}\left(i=1,2, \ldots 2^{k} ; k=1,2,3, \ldots\right)$. Faisons parcoụrir à $h$ une suite de valeurs $h_{k}(k=1,2,3, \ldots)$ qu' on choisit de telle manière que $x+h_{k}$ soit égal à $c_{j}^{(k)}$, c'est à dire $x+h_{k}$ soit le centre d'un intervalle $\delta_{j}^{(k)}$. En choisissant convenablement l'indice $j$, on peut toujours supposer que l'intervalle $\delta_{j}^{(k)}$ ait une extrémité commune avec le segment $p_{i}^{(k)}$ qui contient le point $x$. Il est évident que $h_{b}$ tend vers zéro, car on a

$$
\left|h_{k}\right| \leq \frac{1}{2} \delta_{i}^{(k)}+\rho_{i}^{(k)}=\frac{\varepsilon}{4^{k}}+\frac{1-\varepsilon+\frac{\varepsilon}{2^{k}}}{2^{k}} .
$$

Mais

$$
\frac{\mathfrak{F}\left(x+h_{k}\right)}{h_{k}}=\frac{\mathfrak{F}\left(C_{i}^{(k)}\right)}{h_{k}}=\frac{\varepsilon^{2}}{2^{k}} h_{k} \geq \frac{\varepsilon^{2}}{2^{k}\left(\frac{\varepsilon}{4^{k}}+\frac{1-\varepsilon+\frac{\varepsilon}{2^{k}}}{2^{k}}\right)}=\frac{\varepsilon^{2}}{1-\varepsilon+\frac{\varepsilon}{2^{k}-1}} \geq \varepsilon^{2}
$$

On a donc

$$
\varlimsup_{k \rightarrow 0} \frac{\mathfrak{g}\left(x+h_{k}\right)}{h_{k}} \geq \varepsilon^{2}
$$

Puisque $\varepsilon$ est fixe, cette $\overline{\lim }$ n' est pas nulle, et comme on a

$$
\lim _{k \rightarrow 0} \frac{\mathfrak{F}(x+h)}{h}=0
$$

quand $x+h \subset P$ il en suit que la dérivée $\mathscr{F}^{\prime}(x)$ n' existe pas pour tout point $x$ appartenant à $P$. 
Mais l'ensemble des valeurs de $\mathscr{F}(x)$ sur $P$ contient un seul point, puisque $\mathscr{H}(x)=0$ sur $P$; il suit donc du théorème 5 que $\mathscr{F}(x)$ est une fonction absolument continue de fonction absolument continue, et pourtant elle n'a pas de dérivée sur un ensemble de mesure $1-\varepsilon\left({ }^{1}\right)$.

(c. q. f. d.).

On pourrait construire une fonction absolument continue de fonction abso. lument continue qui n'a même pas de dérivée asymptotique sur un ensemble de mesure $1-\varepsilon$; nous omettons la construction d' un tel exemple.

Nous avons vu qu'une fonction absolument continue de fonction absolument continue peut ne pas avoir de dérivée sur un ensemble dont la mesure est $1-\varepsilon, \varepsilon$ étant aussi petit qu' on veut. Mais la dérivée existe nécessairement sur un ensemble de mesure positive, ce qui est encore un corollaire du théorème 5 .

Corollaire $3\left({ }^{2}\right)$. Une fonction absolument continue de fonction absolument continue a nécessairement une dèrivée délerminee el finie sur un ensemble de mesure positive.

En effet, soit $\mathscr{F}(x)$ uno telle fonction. Supposons que la dérivée $\mathfrak{F}^{\prime}(x)$ n' est déterminée et finie que sur un ensemble $e$ de mesure nulle. L' ensemble $e^{\prime}$ des valeurs de $\mathfrak{F}(x)$ sur $e$ est aussi de mesure nulle puisque $\mathfrak{F}(x)$ jouit de la propriété $N$. Le complémentaire de $e$ par rapport à l'intervalle $(a, b)$ où la fonction $\mathfrak{f}(x)$ est définie est l'ensemble $E$ des point où $\mathscr{F}(x)$ n' existe pas ou n'est pas finie. L'ensemble $\mathcal{E}$ des valeurs de $\mathfrak{F}(x)$ sur $E$ est de mesure nulle en vertu $d u$ théorème 5 . Les valeurs de $\mathfrak{F}(x)$ sur l'intervalle $(a, b)$ appartiennent donc à l'ensemble $e^{\prime}+\mathscr{E}$, et l'on a mes $\left(e^{\prime}+\mathscr{G}\right)=0$. $\mathfrak{F}(x)$ étant continue et l'ensemble de ses valeurs sur $(a, b)$ de mesure nulle, elle est nécessairement constante. Mais dans ce cas la dérivée $\mathfrak{J}(x)$ existe partout, ce qui contredit à l'hypothèse filite au commencement de la démonstration. On voit

(1) M. Ruziewicz, (Remarque à la Note de M. Banach "Su* une classe de fonctions continues ", Fundam. Math., t. 8, 1927, pag. 173), a construit une fonction qui jouit la prepriéte $(S)$ mais n'a pas de dérivée sur un ensemble de meswre positive. Puisque nous savons (\$ 8) que toute fonction vérifiant la condition $(S)$ est une fonction absolument continue de fonction absolument continue (et réciproquement) il en suit que l'exemple de M. Ruzrewicz propve aussi l' existence des fonctions absolument continues de fonctions absolument continues n'ayant pas de dérivée sur un ensemble de mesure positive.

(2) On pourrait deduire ce théorème d' un résultat de $M$. BANACH: toute fonction jouissant de la propriété $(N)$ a une dérivée déterminée et finie sur un ensenable de mesure positive (S. BandCH, Sur une classe de fonctions continues, Fundam. Math. t. 8, 1926, pag. 169). 
ainsi que l'ensemble des points ou la dérivée $\mathfrak{g}^{\prime}(x)$ est déterminée et finie est nécessairement de mesure positive.

(c. q. f. d.).

Corollame 4. Une fonction absolument continue de fonction absolument continue dont la dérivée est nulle presque partout où elle existe se réduit $\dot{a}$ une constante.

En effet, soit $\mathfrak{F}(x)$ cette fonction. Supposons $q u^{\prime}$ elle soit définie dans un intervalle $(a, b)$ et soit, par impossible, $E$ l'ensemble des points où la dérivée $\mathfrak{g}^{\prime}(x)$ n'existe pas ou n'est pas finie. L'ensemble $\mathscr{O}$ des valeurs de $\mathfrak{g}(x)$ sur $E$ est de mesure nulle (théorème 5 ); presque partout sur $C E$ on a par hypothèse $\mathfrak{F}^{\prime}(x)=0$; donc l'ensemble des valeurs de $\mathscr{f}(x)$ sur $C E$ est aussi de mesure nulle en vertu du corollaire 2 du lemme 2 (\$ 7). L' ensemble des valeurs de $\mathscr{f}(x)$ sur $(a, b)$ est donc de mesure nulle et $\mathscr{f}(x)$ se réduit à une constante (l'ensemble $E$ est vide).

(c. q. f. d.).

Il serait pourtant impossible de déduire le corollaire suivant: deux fonctions $\mathscr{F}_{1}(x)$ et $\mathscr{F}_{2}(x)$ dont chacune est une fonction absolument continue de fonctions absolument continue et telles que les dérivées $\mathfrak{F}_{1}^{\prime}(x)$ et $\mathfrak{F}_{2}{ }^{\prime}(x)$ coïncident presque partout où elle existent ne diffèrent que par une constante. Une telle conclusion serait fausse, car la différence des fonctions $\mathfrak{F}_{1}(x)$ et $\mathfrak{F}_{2}(x)$ n' est pas en général une fonction absolument continue de fonction absolument continue. Il est aisé de voir qu' il existe des fonctions absolunent continues de fonctions absolument continues telles que la somme d'une telle fonction et de la variable indépendante $x$ n' est plus une fonction absolument continue de fonction absolument continue.

Pour le voir, reprenons l'ensemble parfait $P$ de mesure $1-\varepsilon$ que nous avons construit et la fonction $\mathscr{f}(x)$ qui n'a pas de dérivée sur $P$. La fonction

$$
\mathfrak{G}_{1}(x)=\mathfrak{F}(x)+x
$$

n'est pas une fonction absolument continue de fonction absolument continue. En effet, en tout point de $P$, la dérivée $\mathfrak{g}^{\prime}(x)$ n' existe pas, celle de $x$ existe, donc celle de $\mathfrak{F}_{1}(x)$ n' existe pas non plus. Mais

$$
\mathfrak{F}_{1}(x)=x
$$

en tout point de $P$, donc l'ensemble des valeurs de $\mathfrak{F}_{1}(x)$ sur $P$ est un ensemble identique à $P$ et sa mesure est égale à $1-\varepsilon$. Il en suit que $\mathfrak{F}_{1}(x)$ n'est pas une fonction absolument continue de fonction absolument continue.

(c. q. f. d.). 
Il est aisé de voir d'ailleurs que $\mathfrak{g}_{:}(x)$ est une intégrale indéfinje de M. M. DEnJoY-KhIn'tchine. C' est ce qu' on déduit immédiatement en remarquant qu' elle est absolument continue dans chaque intervalle contigu à $P$, qu' elle est égale à $x$ en tout point de $P$ et enfin que la série

$$
\sum_{n=1}^{\infty}\left[\mathfrak{F}_{1}\left(b_{n}\right)-\mathfrak{F}_{1}\left(a_{n}\right)\right]=\sum_{n=1}^{\infty}\left(b_{n}-a_{n}\right)=\sum_{n=1}^{\infty} \delta_{n}
$$

$\left[\delta_{n}=\left(a_{n}, b_{n}\right)\right.$ étant un intervalle contign a $\left.P\right]$ est convergente. Cet exemple nous montre donc qu'il exisle des inlégrales indefinies de M. M. DenjoyKhintchine qui ne sont pas des fonctions absolumenl conlinues de fonctions absolument continues.

Nous avous vu que la classe des fonctions absolument continues de fonctions absolument continues contient des fonctions qui n'ont pas de dérivée sur In ensemble de mesure $1-\varepsilon$. Cette classe est done beancoup plus vaste que celle des fonctions absolument continues elles mêmes. Il est naturel de se poser la question s'il est possible d'obtenir une classe encore plus vaste en considérant les fonctions absolument continues des fonctions de la classe étudiée. La réponse est négative en vertu du théorème suivant.

Corolrame 5. Toule fonction de la forme

$$
\mathscr{F}(x)=\psi\{f[\varphi(x)]\}
$$

$\psi, f$ et $\varphi$ etant absolument continues peut êlre prèsentẻe dans la forme

$$
\mathscr{S}(x)=F[\Phi(x)]
$$

les deux fonctions $F$ et $\Phi$ etant encore absolument continues.

En effet, posons

$$
y=\mathscr{F}_{1}(x)=f[\varphi(x) \mid .
$$

Soit $E_{1}$ l'ensemble des points $x$ où la dérivée $\mathfrak{F}_{1}^{\prime}(x)$ n' existe pas ou n' est pas finie, $\mathscr{G}_{1}$ l'onsemble des valeurs de $\mathscr{F}_{1}(x)$ sur $E_{1}$. En vertu du théorème 5 , on a mes $\mathscr{G}_{1}=0$. Soit $R^{\prime}$ l'ensemble de tous les points $y$, ou la dérivée $\psi^{\prime}(y)$ n'existe pas ou n'est pas finie et $R$ l'ensemble de tous les points $x$, tels que les valeurs de $\mathfrak{F}_{1}(x)$ en ces points appartiennent a $R^{\prime}$. On a mes $R^{\prime}=0$, puisque $\psi(y)$ est absolument continue.

Pour tout point $x_{0}$ n'appartenant ui à $R$ ni à $E$, on a

$$
\mathfrak{F}^{\prime}\left(x_{0}\right)=\psi^{\prime}\left(y_{0}\right) \mathfrak{F}_{1}^{\prime}\left(x_{0}\right)
$$


54 M.lle N. Barr et M. D. Menchorf : Sur l' intégrale de Lebesgue-Stieltjes, etc.

la dérivée $\mathfrak{H}^{\prime}\left(x_{0}\right)$ existe donc et elle est finie. L'ensemble $E$ des points $x$ où la dérivée $\mathfrak{g}^{\prime}(x)$ n'existe pas ou n'est pas finie appartient donc à $b_{1}^{\prime}+R$. L'ensemble $\overline{\mathcal{E}}$ des valeurs de $\mathcal{F}(x)$ sur $E$ coincide avec l' ensemble des valeurs de $\psi(y)$ sur $\mathcal{E}$, en désignant par $\mathscr{E}$ l'ensemble des valeurs de $\mathfrak{F}_{1}(x)$ sur $E$. L'ensemble $\mathcal{E}$ est contenu daus la somme $\mathcal{E}_{1} \mid-R^{\prime}$, on a donc mes $\mathcal{G}=0$. En vertu de la continuité absolue de $\psi(y)$, l'ensemble $\overline{\mathcal{G}}$ est donc aussi de mesure nulle et il suit alors du théorème 5 que $\mathfrak{f}(x)$ est une fonction absolument continue de fonction absolument continue.

(c. q. f. d.).

Remarque. On pourrait démontrer le même théorème en tellant compte de la remarque à la fin du théorème 5 . La fonction $\mathfrak{F}_{1}(x)$ peut être présentée dans la forme

$$
\mathfrak{F}_{1}(x)=\vec{f}[\Phi(x)
$$

$\bar{f}$ étant croissanle, et les deux fonctions $\bar{f}$ et $\Phi$ absolument continues; on a alors

$$
\mathscr{F}(x)=\psi_{1}\left[\mathscr{F}_{1}(x)\right]=\psi_{1}\{\tilde{f}[\Phi(x) \mid\}=F[\Phi(x)]
$$

la fonction $F=\psi_{1}(\bar{f})$ est une fonction absolument continue de fonction absolument continue et croissante, elle est donc absolument continue. Le théorème est ainsi démontré. 